Original paper

\title{
Improvement of early detection of breast cancer through collaborative multi-country efforts: Medical physics component
}

\author{
Patricia Mora ${ }^{\mathrm{a}, *}$, Keith Faulkner ${ }^{\mathrm{b}}$, Ahmed M. Mahmoud ${ }^{\mathrm{c}}$, Vesna Gershan ${ }^{\mathrm{d}}$, Aruna Kausik ${ }^{\mathrm{e}}$, \\ Urban Zdesar ${ }^{\mathrm{f}}$, María-Ester Brandan ${ }^{\mathrm{g}}$, Serap Kurt ${ }^{\mathrm{h}}$, Jasna Davidović ${ }^{\mathrm{i}}$, Dina H Salama ${ }^{\mathrm{j}}$, \\ ${\text { Erkin } \text { Aribal }^{\mathrm{k}} \text {, Clara Odio }}^{1}$, Arvind K. Chaturvedi ${ }^{\mathrm{m}}$, Zahida Sabih ${ }^{\mathrm{n}}$, Saša Vujnović ${ }^{\circ}$, Diana Paez $^{\mathrm{p}}$, \\ Harry Delis ${ }^{\mathrm{p}}$
}

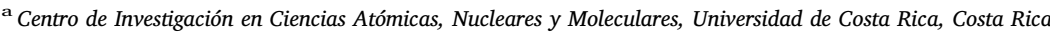

${ }^{\mathrm{b}}$ Hepscott Park, Morpeth, United Kingdom

${ }^{\mathrm{c}}$ Department of Biomedical Engineering and Systems, Cairo University, Egypt

${ }^{\mathrm{d}}$ Faculty of Natural Sciences and Mathematics, Skopje, Macedonia

e Institute of Nuclear Medicine and Allied Sciences, Delhi, India

${ }^{\mathrm{f}}$ DORA Screening Programme, Ljubljana, Slovenia

${ }^{\mathrm{g}}$ Instituto de Física, Universidad Nacional Autónoma de México, 04510 Ciudad de México, Mexico

${ }^{\mathbf{h}}$ Memeder, Istanbul, Turkey

${ }^{\text {i } U n i v e r s i t y ~ C l i n i c a l ~ C e n t r e ~ o f ~ t h e ~ R e p u b l i c ~ o f ~ S r p s k a, ~ D e p a r t m e n t ~ o f ~ M e d i c a l ~ P h y s i c s ~ a n d ~ R a d i a t i o n ~ P r o t e c t i o n, ~ B a n j a ~ L u k a, ~ B o s n i a ~ a n d ~ H e r z e g o v i n a ~}$

${ }^{\mathbf{j}}$ National Center for Radiation Research and Technology, Egyptian Atomic Energy Authority, Egypt

${ }^{\mathbf{k}}$ Acibadem University, Radiology Department, Altunizade Hospital, Breast Health Center, Istanbul Turkey

${ }^{1}$ Departamento de Radiología, Hospital Max Peralta, Costa Rica

${ }^{m}$ Rajiv Gandhi Cancer Institute and Research Centre, Delhi, India

${ }^{n}$ Multan Institute of Nuclear Medicine and Radiotherapy, Multan, Pakistan

${ }^{\circ}$ University Clinical Centre of the Republic of Srpska, Department of Clinical Radiology, Banja Luka, Bosnia and Herzegovina

P International Atomic Energy Agency, Vienna, Austria

\section{A R T I C L E I N F O}

\section{Keywords:}

IAEA

Mammography

Breast cancer

Quality control

\begin{abstract}
A B S T R A C T
Purpose: The International Atomic Energy Agency (IAEA) through a Coordinated Research Project on "Enhancing Capacity for Early Detection and Diagnosis of Breast Cancer through Imaging", brought together a group of mammography radiologists, medical physicists and radiographers; to investigate current practices and improve procedures for the early detection of breast cancer by strengthening both the clinical and medical physics components. This paper addresses the medical physics component.

Methods: The countries that participated in the CRP were Bosnia and Herzegovina, Costa Rica, Egypt, India, Kenya, the Frmr. Yug. Rep. of Macedonia, Mexico, Nigeria, Pakistan, Philippines, Slovenia, Turkey, Uganda, United Kingdom and Zambia. Ten institutions participated using IAEA quality control protocols in 9 digital and 3 analogue mammography equipment. A spreadsheet for data collection was generated and distributed. Evaluation of image quality was done using TOR MAX and DMAM2 Gold phantoms.

Results: QC results for analogue equipment showed satisfactory results. QC tests performed on digital systems showed that improvements needed to be implemented, especially in thickness accuracy, signal difference to noise ratio (SDNR) values for achievable levels, uniformity and modulation transfer function (MTF). Mean glandular dose (MGD) was below international recommended levels for patient radiation protection. Evaluation of image quality by phantoms also indicated the need for improvement.

Conclusions: Common activities facilitated improvement in mammography practice, including training of medical physicists in QC programs and infrastructure was improved and strengthened; networking among medical physicists and radiologists took place and was maintained over time. IAEA QC protocols provided a uniformed approach to QC measurements.
\end{abstract}

\footnotetext{
* Corresponding author.

E-mail address: patricia.mora@ucr.ac.cr (P. Mora).
} 


\section{Introduction}

In 2012, there were an estimated 1670000 cases of breast cancer diagnosed worldwide [1] making breast cancer the second most common cancer in the world and the most common cancer in women. According to the International Agency for Research on Cancer (IARC), over half of breast cancers occurred in the less developed countries. Incidence rates vary between regions, with the lowest rate in Central Africa (27/100000) and the highest in Belgium (111,9/100000). Deaths from breast cancer are disproportionately higher in less developed countries with $62 \%$ of total deaths occurring in these countries. As countries develop breast screening programs it is likely that breast cancer incidence rates will increase due to its early detection.

At the moment, X-ray mammography is the only technique that has proven the ability to detect breast cancer at an early stage, before the cancer is palpable and is the basis of the most organized breast screening programs to detect breast cancer in a non-symptomatic population. For mammography to be effective at detecting breast cancer at an early stage, adequate differentiation of small masses and microcalcifications is required, which in principle can only produce subtle contrast differences in mammography images. These imaging requirements place high technical demands on the imaging equipment and require high image quality and rigorous Quality Assurance (QA) to maintain these standards. Additional constraints are placed as the radiation dose should be kept at the lowest possible level, given the size average breast composition or average breast glandularity of the nonsymptomatic target group, and the radiosensitivity of the breast. Routine performance testing of mammography imaging equipment by competent medical physicists is an essential component of a comprehensive QA program for mammography screening [2,3]. Additionally, it is apparent that all involved medical professionals have to be properly trained and highly acquainted with the mammographic procedure.

In 2012, the International Atomic Energy Agency (IAEA) started a Coordinated Research Project (CRP): IAEA CRP E1.30.39 Enhancing Capacity for Early Detection and Diagnosis of Breast Cancer through Imaging, which grouped together mammography radiologists, medical physicists and radiographers from 15 different countries. During the 4year period of the project many activities were undertaken with the intention to investigate current practices, aiming to improve early detection of breast cancer by strengthening both the clinical and medical physics components. In this context, as part of the CRP activities participants received additional training in several components of the QA process. The overall objective of the CRP, from the Medical Physics perspective, was to contribute to the improvement in diagnosis and detection of breast cancer following the application of international standards of best practice in mammography. The CRP activities were specifically designed to: familiarize participants with the assessment of image quality and dosimetry requirements for mammography; training medical physicists and radiographers on performing measurements and collecting data, improving the provision of QA processes; collecting comprehensive Quality Control (QC) results in participating institutions using the relevant IAEA protocols; analyzing and evaluating these QC results and comparing them with internationally established requirements and tolerances for corrective actions $[2,4]$; evaluating image quality in mammography units in a standardized way, using a common phantom and centralized analysis of the images, and finally create a network of medical physicists and radiologists that can support each other on the technical aspects of mammography.

There are a number of differences between the IAEA protocol and that of the European protocol. The main difference is that in the IAEA approach, measurement of contrast detail performance uses the same test object, but with an automated reading system, which is how many individuals apply the EC test object/protocol these days. In addition, there are slight differences in the specification of the position for the test object for assessment of SNR values, but which would not cause there to be a significant difference between the IAEA protocol and that of the EC. The main difference is that the specifications for MTF measurements is more detailed in the IAEA protocol than in the EC's one.

\section{Materials and methods}

\subsection{Participants}

Countries participating in the IAEA CRP had very different levels of implementation of their breast screening programs and large deviations in the available mammography equipment and corresponding conformance with established quality assurance programs. To remove this local bias in terms of the level of QA implementation, and standardize the practices, all groups followed a common methodology for the QC test. IAEA Human Health Series No.2 [4] and No.17 [2] were agreed as references (for screen-film and digital mammography, respectively), allowing uniform collections of basic QC metrics and assessment of the performance of participating mammography equipment.

A total of 15 countries participated in different phases of the CRP (Bosnia and Herzegovina, Costa Rica, Egypt, India, Kenya, the Frmr. Yug. Rep. of Macedonia, México, Nigeria, Pakistan, Philippines, Slovenia, Turkey, Uganda, United Kingdom, Zambia), whereas 9 agreed to take part in this equipment testing inter-comparison. Table 1 shows information about participating institutes in terms of mammography equipment (analogue/digital), institution, existence of established screening program at the commencement of the CRP, number of clinical procedures during 2015 and implementation of organized QC programs before and after this CRP. Analogue equipment with its screen/film combination that participated in the study were: Planmed Nuance Classic (CAWO MAMMO R200/Kodak MIN R), Siemens Balance (Kodak MIN R 2000/AGFA HT) and Metaltronica Flat SE (Agfa HD Mamoray/

Table 1

Participating countries, institutions and procedures.

\begin{tabular}{|c|c|c|c|c|c|c|c|c|c|}
\hline Country & A & $\mathrm{D}$ & Institution/City & SC & Tomo & Bio & Procedures & QC Before & QC After \\
\hline Bosnia and Herzegovina & & 1 & University Clinical Centre of the Republic of Srpska/Banja Luka & $\mathrm{O}$ & $\mathrm{N}$ & $\mathrm{Y}$ & 3168 & $\mathrm{Y}$ & $\mathrm{Y}$ \\
\hline Costa Rica & 1 & 1 & Hospital Max Peralta/Cartago & $\mathrm{O}$ & $\mathrm{N}$ & $\mathrm{N}$ & 11882 & $\mathrm{~N}$ & $\mathrm{Y}$ \\
\hline Egypt & & 1 & Women and Fetal Imaging Centre/Cairo & $\mathrm{O}$ & $\mathrm{N}$ & $\mathrm{N}$ & 1403 & $\mathrm{~N}$ & $\mathrm{Y}$ \\
\hline India A & & 1 & Rajiv Gandhi Cancer Institute and Research Centre/Delhi & $\mathrm{Y}$ & $\mathrm{Y}$ & $\mathrm{Y}$ & 1760 & $\mathrm{Y}$ & $\mathrm{Y}$ \\
\hline India B & & 1 & Institute of Nuclear Medicine and Allied Sciences/Delhi & $\mathrm{Y}$ & $\mathrm{N}$ & $\mathrm{Y}$ & 2174 & $\mathrm{Y}$ & $\mathrm{Y}$ \\
\hline Macedonia & 1 & 1 & Faculty of Natural Sciences and Mathematics/Skopje & Y & $\mathrm{N}$ & $\mathrm{N}$ & 1560 & $\mathrm{~N}$ & $\mathrm{Y}$ \\
\hline México & & 1 & Universidad Nacional Autónoma de México/Cuidad de México & * & $\mathrm{N}$ & $\mathrm{N}$ & - & $\mathrm{Y}$ & $\mathrm{Y}$ \\
\hline Pakistan & 1 & & Multan Institute of Nuclear Medicine and Radiotherapy/Multan & $\mathrm{Y}$ & $\mathrm{N}$ & $\mathrm{Y}$ & 1700 & $\mathrm{Y}$ & $\mathrm{Y}$ \\
\hline Slovenia & & 1 & DORA Screening Programme/Ljubljana & $\mathrm{Y}$ & $\mathrm{N}$ & $\mathrm{N}$ & 39745 & $\mathrm{Y}$ & $\mathrm{Y}$ \\
\hline Turkey & & 1 & Memeder, Istanbul & $\mathrm{Y}$ & $\mathrm{N}$ & $\mathrm{Y}$ & 3549 & $\mathrm{~N}$ & $\mathrm{Y}$ \\
\hline TOTAL & 3 & 9 & & 6 & 1 & 5 & & 6 & 10 \\
\hline
\end{tabular}

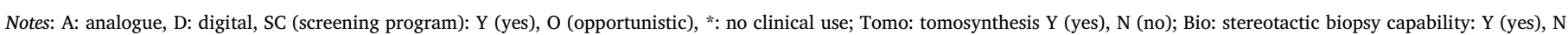
(no); Procedures: number of procedures done during 2015; QC Before: QC implemented before intervention and QC After: QC implemented after intervention. 
Agfa Mamoray HDR \& Fuji AD-M or KODAK Min R 2190/Fuji AD-M \& Agfa Mamoray HDR). No Computed Radiology equipment participated in this study. Digital equipment (FFDM) that was studied: GE Senographe Essential, GE Senographe 2000D, Siemens Inspiration, Hologic Selenia and FUJI Amulet s.

\subsection{Testing protocols}

In order to harmonize the application of the testing protocols, training was provided to the associated staff during a CRP meeting in Slovenia in November 2013, where medical physicists, radiographers and radiologists were present. During a review meeting in Vienna in 2016 the results obtained by the participants were reviewed and discussed, and the common spreadsheet was also reviewed and fine-tuned.

To demonstrate the process and the benefit of the CRP in the clinical practice, relevant QC tests both for analogue and digital equipment were agreed to be performed within each institution twice; one in the early phases of the CRP and one near completion.

Participating institutes with analogue equipment were asked to submit information for the following:

- Kilovoltage accuracy and radiation output at $28 \mathrm{kVp}$,

- Automated exposure control (AEC) performance,

- Half value layer (HVL at $28 \mathrm{kVp}$ ),

- Mean glandular dose (MGD) and

- Film processor sensitometry.

Since many countries were new to digital QC, and in order to standardize the data collection process, the University of Costa Rica (UCR) put together a spreadsheet for data collection based on the IAEA methodology [2]. By means of built-in macros, the spreadsheet automatically checks for tolerances and limiting values, facilitating easy evaluation of performance and compliance of the CRP participants. The CRP team created a group in a popular mobile application, allowing almost real-time support and guidance to the participants, led by the medical physics personnel from the UCR.

The spreadsheet, that automatically generates a printable report with the main results, covered the following tests, (shown in parenthesis are the sections from the protocol):

- Unit assembly (8.2.1),

- Geometry: compression force and thickness accuracy indicator (8.3.1) and collimation system (8.9),

- X Ray tube: half value layer (8.7.1),

- Automatic exposure control performance: SNR and SDNR (8.4),

- Image quality: spatial linearity and geometric distortion (8.5.3), ghosting (8.5.4), uniformity and artefact evaluation (8.5.5) and modulation transfer function (8.6.1),

- Dosimetry: mean glandular dose (8.8.1)

Main points to mention are: test object for SDNR was a $0,2 \mathrm{~mm}$ thick square of aluminium of $10 \mathrm{~mm}$ on a side placed on a $20 \mathrm{~mm}$ PMMA slab, MTF test tool was placed on top of $45 \mathrm{~mm}$ PMMA and for dosimetry mean glandular dose is obtained from incident kerma in air using the formula used in Europe and United Kingdom with variable glandular content. Detector response function and noise characteristics were not evaluated in this study.

\subsection{Image quality assessment}

For the evaluation of the image quality, two different types of comprehensive phantoms were procured by the IAEA and distributed to all participants under the framework of CRP project, depending on the available equipment:

Analogue equipment: the TOR MAX phantom (Fig. 1) by Leeds Test Objects [5], was utilized for the evaluation of sensitometry (ten-step

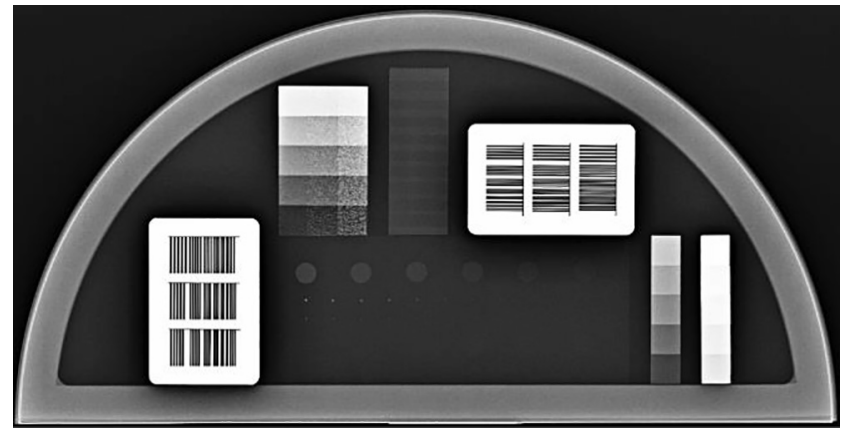

Fig. 1. Image of TOR MAX phantom.

grey-scale), high contrast resolution ( 1,0 to $20,0 \mathrm{lp} / \mathrm{mm})$, low contrast resolution $(1,8-51 \mathrm{p} / \mathrm{mm})$, low-contrast large-detail detectability (12 details, 5,6 mm diameter) and high-contrast small-detail detectability (11 objects, $0,5 \mathrm{~mm}$ and $0,25 \mathrm{~mm}$ diameter); analysis of the TOR MAX image was performed by local physicists.

Digital equipment: image quality for digital units is commonly defined in terms of the threshold detectability of small gold discs within a contrast-detail mammography phantom, such as the CDMAM [6] used in EUREF protocols [7]. In this research project the DMAM2 Gold phantom was used [8] (Fig. 2) containing 78 threshold contrast details in 6 sizes, with diameters ranging from $0,1 \mathrm{~mm}$ to $2,0 \mathrm{~mm}$ and with contrast range from $0,49 \%$ to $27,56 \%$. This phantom also meets the standards for the European Guidelines for quality assurance in breast cancer screening and diagnosis [3].

To avoid ambiguity in the analysis of the images generated by the participants, DMAM2 Gold images were analysed centrally at the UCR using a dedicated software provided by the phantom vendor, AutoPIA (Automatic Phantom Image Analysis) by Cyberqual Srl (Gorizia, IT), which automatically localizes phantom details [9]. Software validation has been documented [10].

The phantom was placed between two slabs of $20 \mathrm{~mm}$ PMMA and total thickness was $53 \mathrm{~mm}$. Exposure for each unit was made with automatic exposure control (technique factors for each equipment are listed on Table 2). A total of 16 "raw" (or "unprocessed") images, were collected for each equipment and sent to the UCR for AutoPIA software analysis.

Once the software automatically recognizes the phantom type, it localizes the groups of details within the image and the individual details within each group. For each detail, the image quality is evaluated utilizing the contrast-to-noise ratio (CNR) defined, according to the Rose's model, as the signal difference between the detail of interest and the surrounding background, divided by the noise of the background [11]. For "large-area" details, the software calculates the CNR based on a circular ROI inside the detail from which it calculates the mean pixel value to get the detail signal, then with the use of a circular ring surrounding the detail, it calculates from it the mean pixel value and standard deviation to get the background signal and noise, respectively. For "small details" $(0,5 \mathrm{~mm}$ and $0,25 \mathrm{~mm})$ the signal produced by the detail is calculated by weighting the individual differences between the pixel value of each pixel belonging to the detail and the surrounding background [12].

Analysis of the modulation transfer function (MTF) has also been carried out centrally by UCR medical physicists, using ImageJ plug in for performing physical characterization and quality checks known as "COQ" $[15,16]$.

\subsection{Dosimetry}

To assess the mean glandular dose (MGD), the methodology of Dance et al. [17] was utilized, as described in IAEA publications $[2,13,14]$ using the following equation: 


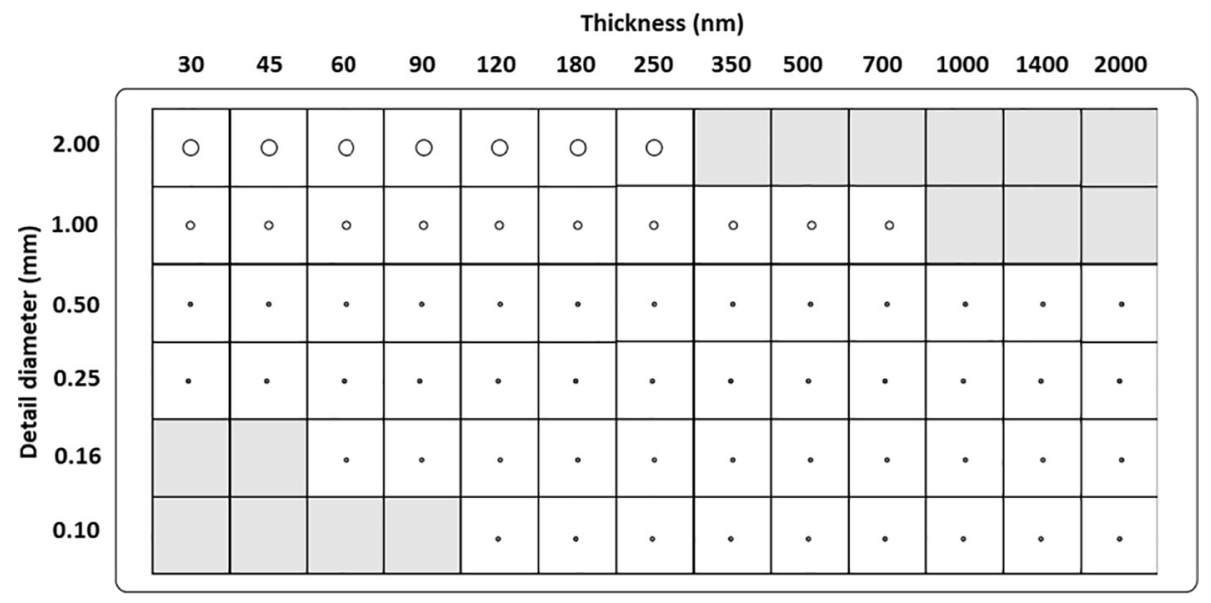

Fig. 2. Layout of the contrast detail phantom DMAM2 Gold.

Table 2

Technical parameters for image quality evaluation.

\begin{tabular}{|c|c|c|c|c|c|}
\hline \multirow[t]{2}{*}{ Type } & \multirow[t]{2}{*}{ Equipment ID } & \multicolumn{4}{|c|}{$\begin{array}{l}\text { Technical parameters for image quality evaluation } \\
\text { (DMAM2 Gold or TOR MAX) }\end{array}$} \\
\hline & & $\mathrm{kVp}$ & $\begin{array}{l}\text { Anode/ } \\
\text { Filter }\end{array}$ & $\begin{array}{l}\text { Incident air } \\
\text { Kerma (mGy) }\end{array}$ & $\begin{array}{l}\text { Thickness } \\
(\mathrm{mm})\end{array}$ \\
\hline \multirow[t]{9}{*}{ FFDM } & D1 & 30 & $\mathrm{Rh} / \mathrm{Rh}$ & 5,59 & 57 \\
\hline & D2 & 31 & $\mathrm{Rh} / \mathrm{Rh}$ & 4,77 & 53 \\
\hline & D3 & 32 & $\mathrm{~W} / \mathrm{Rh}$ & 5,57 & 66 \\
\hline & D4 & 31 & $\mathrm{~W} / \mathrm{Rh}$ & 7,46 & 60 \\
\hline & D5 & 32 & $\mathrm{~W} / \mathrm{Rh}$ & 6,47 & 66 \\
\hline & D6 & 30 & $\mathrm{~W} / \mathrm{Rh}$ & 5,84 & 59 \\
\hline & D7 & 32 & $\mathrm{~W} / \mathrm{Rh}$ & 6,47 & 66 \\
\hline & D8 & 29 & $\mathrm{~W} / \mathrm{Rh}$ & 6,12 & 52 \\
\hline & D9 & 30 & $\mathrm{~W} / \mathrm{Rh}$ & 3,72 & - \\
\hline \multirow[t]{3}{*}{ Analogue } & $\mathrm{A} 1$ & 28 & Mo/Mo & - & - \\
\hline & A2 & 28 & Mo/Mo & - & - \\
\hline & A3 & 28 & Mo/Mo & - & - \\
\hline
\end{tabular}

$M G D=\mathrm{g}_{t} c_{t} \mathrm{~s} K_{i, t}$

where, $\mathrm{K}_{\mathrm{i}, \mathrm{t}}$ is the incident air kerma at each PMMA phantom thickness, the $g_{t}$-factor converts air kerma to MGD for a breast of glandularity of $50 \%$ and the $c_{t}$-factor corrects for the difference in breast composition from $50 \%$ glandularity and finally, $s$ is the factor which corrects for the target filter combination of the X-ray tube.

\section{Results and discussion}

Table 3, shows the results for the 3 analogue mammography equipment from the annual QC test for 2013 and 2015. Facilities reported implementation of sensitometry QC; general parameters like $\mathrm{kVp}$ accuracy, output and HVL were within recommended tolerances in all systems. MGD was also below the recommended 2,5 mGy value for 45 mm PMMA in all systems, except in system A1 in 2015.

Tables 4a, 4b and 4c; show the initial results for the QC metrics that allowed the CRP group to evaluate the overall performance of the 9 digital mammography systems that participated in this study. It should be noted that the system D8 failed the powered system compression force, and systems D6 and D7, which came from the same manufacturer, failed the thickness accuracy. For the AEC, all systems passed recommended acceptable values for signal difference to noise ratio (SDNR) for 20, 45 and $70 \mathrm{~mm}$ PMMA, except for D1 and D8 at the largest phantom thickness (Table 4a). Regarding achievable SDNR values, D1 failed for 20, 45 and $70 \mathrm{~mm}$ PMMA, D2 failed for $45 \mathrm{~mm}$ PMMA, D5 and D6 failed for $70 \mathrm{~mm}$ PMMA and D8 failed for 45 and $70 \mathrm{~mm}$ PMMA (Table 4b). It should be noted that system D2, does not
Table 3

QC results for analogue equipment.

\begin{tabular}{|c|c|c|c|c|c|c|c|}
\hline \multirow{3}{*}{\multicolumn{2}{|c|}{ QC Test }} & \multicolumn{6}{|c|}{ Analogue Equipment } \\
\hline & & \multicolumn{2}{|l|}{$\mathrm{A} 1$} & \multicolumn{2}{|l|}{ A2 } & \multicolumn{2}{|l|}{ A3 } \\
\hline & & 2013 & 2015 & 2013 & 2015 & 2013 & 2015 \\
\hline \multicolumn{2}{|c|}{$\begin{array}{l}\text { Measured } \mathrm{kVp} \text { at } 28 \mathrm{kVp} \text { nominal } \\
\quad(\mathrm{Mo} / \mathrm{Mo})\end{array}$} & 28,10 & 27,90 & 28,30 & 28,28 & 28,56 & 28,56 \\
\hline \multicolumn{2}{|c|}{$\begin{array}{l}\text { Output at } 28 \mathrm{kVp}(1 \mathrm{~m}) \mathrm{Mo} / \mathrm{Mo} \\
\qquad(\mu \mathrm{Gy} / \mathrm{mAs})\end{array}$} & 33,40 & 34,04 & 63,25 & 62,34 & 48,00 & 50,00 \\
\hline AEC $20 \mathrm{~mm}$ & mAs & 15,70 & 15 & 6,10 & 6,80 & n.a & 15 \\
\hline $\begin{array}{l}\text { PMMA, } \\
28 \mathrm{kVp}\end{array}$ & OD & 2,04 & 1,27 & 1,39 & 1,35 & n.a & 1,65 \\
\hline AEC $45 \mathrm{~mm}$ & mAs & 63,30 & 105 & 49,1 & 52,3 & 90 & 100 \\
\hline $\begin{array}{l}\text { PMMA, } \\
28 \mathrm{kVp}\end{array}$ & OD & 2,10 & 1,57 & 1,61 & 1,30 & 1,70 & 1,80 \\
\hline AEC $70 \mathrm{~mm}$ & mAs & 107 & 519 & 267 & 284,20 & n.a & 600 \\
\hline $\begin{array}{l}\text { PMMA, } \\
28 \mathrm{kVp}\end{array}$ & OD & 2,05 & 1,65 & 1,50 & 1,18 & n.a & 1,90 \\
\hline \multicolumn{2}{|c|}{$\mathrm{HVL}$ at $28 \mathrm{kVp} \mathrm{Mo} / \mathrm{Mo}(\mathrm{mmAl})$} & 0,35 & 0,35 & 0,32 & 0,30 & 0,36 & 0,37 \\
\hline \multicolumn{2}{|c|}{ Ki 45 mm PMMA Mo/Mo (mGy) } & 7,40 & 14,80 & 7,91 & 8,28 & 6 & 6,20 \\
\hline \multicolumn{2}{|c|}{$\begin{array}{l}\text { MGD } 45 \text { mm PMMA Mo/Mo } \\
\text { (mGy) }\end{array}$} & 1,38 & 2,85 & 1,73 & 1,80 & 1 & 1 \\
\hline \multirow[t]{3}{*}{ TOR MAX } & $\mathrm{lp} / \mathrm{mm}$ & 15 & 15 & 13 & 13 & n.a & 15 \\
\hline & $\begin{array}{l}\text { Number of } \\
\text { disc }\end{array}$ & 7 & 7 & 7 & 7 & n.a & 6 \\
\hline & OD & 1,71 & 1,65 & 1,58 & 1,48 & n.a & 1,65 \\
\hline \multicolumn{2}{|c|}{ Processor QC (Yes/No) } & Yes & Yes & Yes & Yes & Yes & Yes \\
\hline \multicolumn{2}{|c|}{ Mean Gradient of film } & n.a & 1,79 & n.a & 2,70 & n.a & 2,85 \\
\hline
\end{tabular}

Note: n.a: not available.

meet the standard for some indices, is not being used clinically.

Fig. 3, shows for each digital equipment the MGD for equivalent breast thickness to 20, 45 and $70 \mathrm{~mm}$ of PMMA phantom. The international recommended acceptable and achievable levels in mGy are as follows: for $20 \mathrm{~mm}, 1,0 / 0,6$; for $45 \mathrm{~mm}, 2,5 / 2,0$; and for $70 \mathrm{~mm}, 6,5$ / 5,1 . [2]. Those recommended levels have been derived from screen-film mammography, being a common issue to existing mammography protocols. Therefore, even though the results show an overall good compliance with international reference values, the dose values for thicker breast are unexpectedly low for the majority of the systems thereby potentially compromising image quality, as also demonstrated by the fact that D5, D6 and D8 fail the achievable SDNR at the highest breast thicknesses.

In Fig. 4, the Figure of Merit (FOM), as the ratio of SDNR squared divided by MGD, permits a quantitative assessment of the overall performance in terms of image quality and patient doses [18].

The spatial resolution properties of participating digital imaging systems are shown by means of the MTF graphs in Fig. 5. COQ Plug in 
Table 4a

QC results for digital equipment.

\begin{tabular}{|c|c|c|c|c|c|c|c|c|c|}
\hline \multirow[t]{2}{*}{ Equipment ID } & \multirow[t]{2}{*}{ Unit assembly } & \multicolumn{2}{|l|}{ Compression force $(\mathrm{N})$} & \multicolumn{3}{|c|}{ Measured compression thickness (mm) } & \multicolumn{3}{|c|}{ SDNR values (Acceptable/Achievable level) } \\
\hline & & Powered & Manual & $20 \mathrm{~mm}$ & $45 \mathrm{~mm}$ & $70 \mathrm{~mm}$ & $20 \mathrm{~mm}$ & $45 \mathrm{~mm}$ & $70 \mathrm{~mm}$ \\
\hline D1 & $\sqrt{ }$ & 194 & 292 & 24 & 50 & 75 & 15,38/Fail & 13,86/Fail & $5,26^{*} /$ Fail \\
\hline D2 & $\checkmark$ & 170 & 180 & 19 & 44 & 70 & $14,07 / \mathrm{V}$ & 10,35/Fail & n.a \\
\hline D3 & $\sqrt{ }$ & 130 & 125 & 23 & 48 & 74 & $8,67 / V$ & $7,74 / v$ & $7,06 / v$ \\
\hline D4 & $\sqrt{ }$ & 200 & 200 & 21 & 45 & 72 & $9,75 / V$ & $8,08 / \mathrm{V}$ & $5,65 / \sqrt{ }$ \\
\hline D5 & $\checkmark$ & 188 & 124 & 20 & 46 & 71 & $9,38 / \mathrm{V}$ & $7,25 / v$ & 4,63/Fail \\
\hline D6 & $\checkmark$ & 180 & 183 & 20 & 45 & 70 & $10,02 / V$ & $7,41 / V$ & 3,86/Fail \\
\hline D7 & $\checkmark$ & 164 & 258 & $32^{*}$ & $56^{*}$ & $82^{*}$ & $10,15 / V$ & $7,55 / \sqrt{ }$ & $5,67 / V$ \\
\hline D8 & $\checkmark$ & $143^{*}$ & 161 & 18 & 42 & 69 & $10,73 / V$ & 6,26/Fail & $1,43^{*} /$ Fail \\
\hline D9 & $\checkmark$ & $140^{*}$ & 150 & 21 & 45 & 70 & $15,77 / \mathrm{V}$ & $12,27 / V$ & $9,70 / \mathrm{V}$ \\
\hline
\end{tabular}

Note: * means value is outside tolerance. For AEC first number is the value, check and fail refer to if this value passes the tolerance at the achievable level.

Table 4b

$\mathrm{QC}$ results for digital equipment.

\begin{tabular}{|c|c|c|c|c|c|c|c|c|}
\hline \multirow[t]{2}{*}{ Equipment ID } & \multicolumn{2}{|c|}{ Spatial Linearity and Geometric Distortion } & \multicolumn{2}{|c|}{ Detector Ghosting } & \multirow[t]{2}{*}{ Uniformity } & \multicolumn{3}{|c|}{$\begin{array}{l}\text { HVL for AEC setting at different PMMA } \\
(\mathrm{mmAl})\end{array}$} \\
\hline & Visual Test & Known Distances & SDNR & Observable & & $20 \mathrm{~mm}$ & $45 \mathrm{~mm}$ & $70 \mathrm{~mm}$ \\
\hline D1 & $\checkmark$ & $\sqrt{ }$ & n.a & n.a & n.a & 0,36 & 0,45 & 0,46 \\
\hline D2 & $\checkmark$ & $\checkmark$ & 0,41 & Yes* $^{*}$ & Fail & 0,34 & 0,42 & n.a \\
\hline D3 & $\sqrt{ }$ & $\sqrt{ }$ & 0,56 & No & $\sqrt{ }$ & 0,50 & 0,53 & 0,57 \\
\hline D4 & $\checkmark$ & n.a & 0,20 & No & $\checkmark$ & 0,50 & 0,54 & 0,57 \\
\hline D5 & V & v & 0,08 & No & $\checkmark$ & 0,41 & 0,43 & 0,44 \\
\hline D6 & $\checkmark$ & V & 0,14 & No & V & 0,50 & 0,53 & 0,54 \\
\hline D7 & $\checkmark$ & V & 0,23 & No & V & 0,48 & 0,51 & 0,57 \\
\hline D8 & $\checkmark$ & $V$ & 0,02 & No & V & $0,27^{*}$ & $0,29^{*}$ & 0,45 \\
\hline D9 & V & V & 0,67 & No & $V$ & 0,53 & 0,57 & 0,60 \\
\hline
\end{tabular}

Note: * means value is outside tolerance.

Table 4c

$\mathrm{QC}$ results for digital equipment.

\begin{tabular}{|c|c|c|c|c|c|c|c|c|}
\hline \multirow[t]{2}{*}{ Equipment ID } & \multicolumn{3}{|c|}{ MGD (mGy) (Acceptable/Achievable level) } & \multicolumn{3}{|c|}{ Collimation for $24 \mathrm{~cm} \times 30 \mathrm{~cm}$} & \multicolumn{2}{|l|}{ MTF } \\
\hline & $20 \mathrm{~mm}$ & $45 \mathrm{~mm}$ & $70 \mathrm{~mm}$ & $\begin{array}{l}\text { Light Field Detector } \\
\text { Congruence }\end{array}$ & $\begin{array}{l}\text { Missed Tissue } \\
\text { Measurement } \\
(\mathrm{mm})\end{array}$ & $\begin{array}{l}\text { Chestwall Edge-Compression Paddle } \\
\text { Diference } \\
(\mathrm{mm})\end{array}$ & $50 \%$ & $20 \%$ \\
\hline D1 & $0,95 / \mathrm{V}$ & $1,37 / \mathrm{V}$ & $1,77 / \sqrt{ }$ & n.a & n.a & n.a & $2,18^{*}$ & $4,27^{*}$ \\
\hline $\mathrm{D} 2^{*}$ & $0,53 / \sqrt{ }$ & $1,37 / \sqrt{ }$ & n.a & n.a & n.a & n.a & 3,80 & 5,31 \\
\hline D3 & $0,51 / V$ & $1,23 / \mathrm{V}$ & $3,43 / \mathrm{V}$ & $\sqrt{ }$ & 3 & 2 & 5.48 & n.a \\
\hline D4 & $0,53 / \sqrt{ }$ & $1,30 / \mathrm{V}$ & $2,79 / \sqrt{ }$ & $\sqrt{ }$ & 3 & 0 & $4,79^{*}$ & n.a \\
\hline D5 & $0,63 / \sqrt{ }$ & $1,24 / \sqrt{ }$ & $1,19 / \mathrm{V}$ & V & 2,4 & 1,9 & $3,86^{*}$ & n.a \\
\hline D6 & $0,60 / v$ & $1,35 / \mathrm{V}$ & $1,20 / V$ & $\sqrt{ }$ & 5 & 0 & 4,21 & n.a \\
\hline D7 & $0,60 / \sqrt{ }$ & $1,48 / V$ & $1,62 / \mathrm{V}$ & $\checkmark$ & 5 & 0 & n.a & n.a \\
\hline D8 & $1,13^{*} / V$ & $1,52 / \mathrm{V}$ & $1,64 / \mathrm{V}$ & $\checkmark$ & 2 & 0 & n.a & n.a \\
\hline D9 & $0,59 / \sqrt{ }$ & $1,28 / v$ & $2,87 / V$ & V & n.a & n.a & 5,2 & 9,42 \\
\hline
\end{tabular}

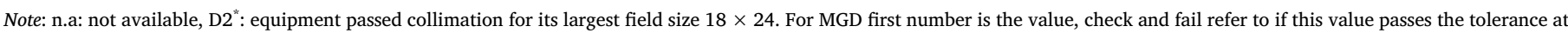
the achievable level.

for ImageJ, does not calculate further to the cut-off (Nyquist) frequency, not allowing determination of MTF at 20\%, for units D3 and D4 both from same manufacturer. Most systems did not pass the recommended values given in Human Health No.17 publication, this ilustrates one of the weaknesses in using MTF as a QA measure.

Finally, image quality based on threshold contrast visibility was evaluated with the DMAM2 Gold phantom and software. The last visible detail identified by the software gave the gold thickness associated for this detail, which was then compared with the acceptable and achievable values of section 2b.2.4 from European guidelines [3]. Fig. 6, shows for each equipment the performance for objects of different sizes. The squares filled with green colour show until which value the system was able to resolve. The acceptable and achievable values are marked with solid and dash lines respectively. Results show that only systems D2, D3, D4 and D9 performed according to the acceptable value of threshold contrast visibility. Optimization of image quality can be implemented in all equipment since very few have green squares that run until the dash line (achievable value). It is to be noted that for the object with diameter 0,5; equipment D1, D5, D6, D7 and D8 were not able to meet the standard for this size but were able to show better results for smallest objects.

After the presented results of the QC test were discussed among all participants and individual efforts and corrective actions were implemented in different facilities. The following list gives an overview of the main interventions that resulted from this common exercise.

- One of the analogue equipment with poor performance in this study was replaced by a digital one (not included in this paper). Additional training activities were organized with the active support of the radiologists and through IAEA assistance. Four medical physicists 


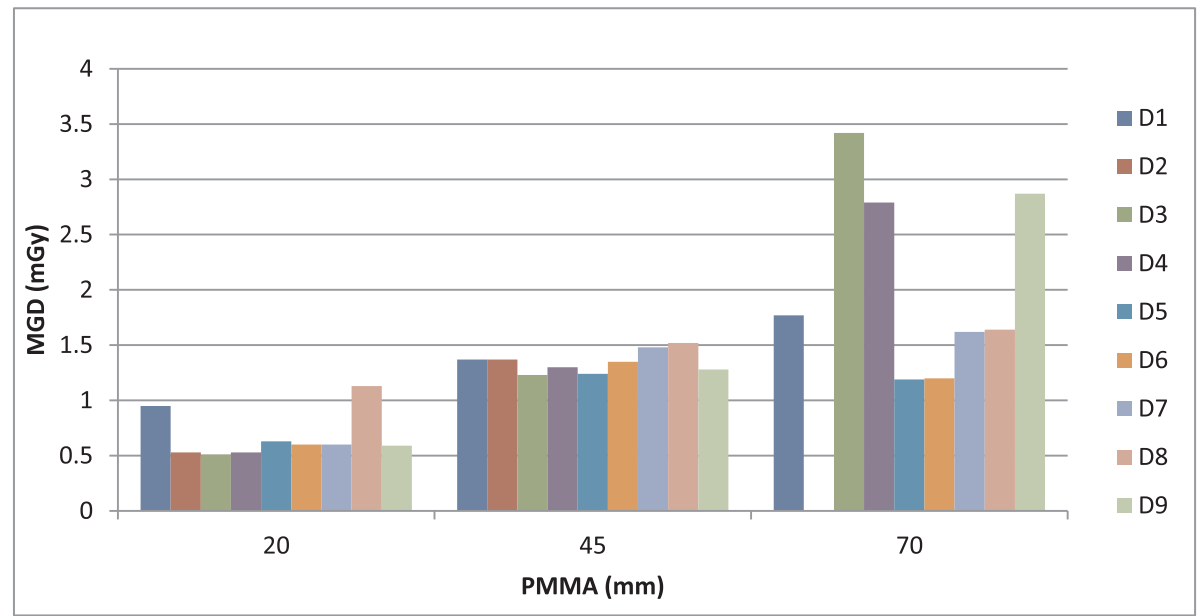

Fig. 3. Mean glandular dose (mGy) for 20,45 and 70 mm PMMA.

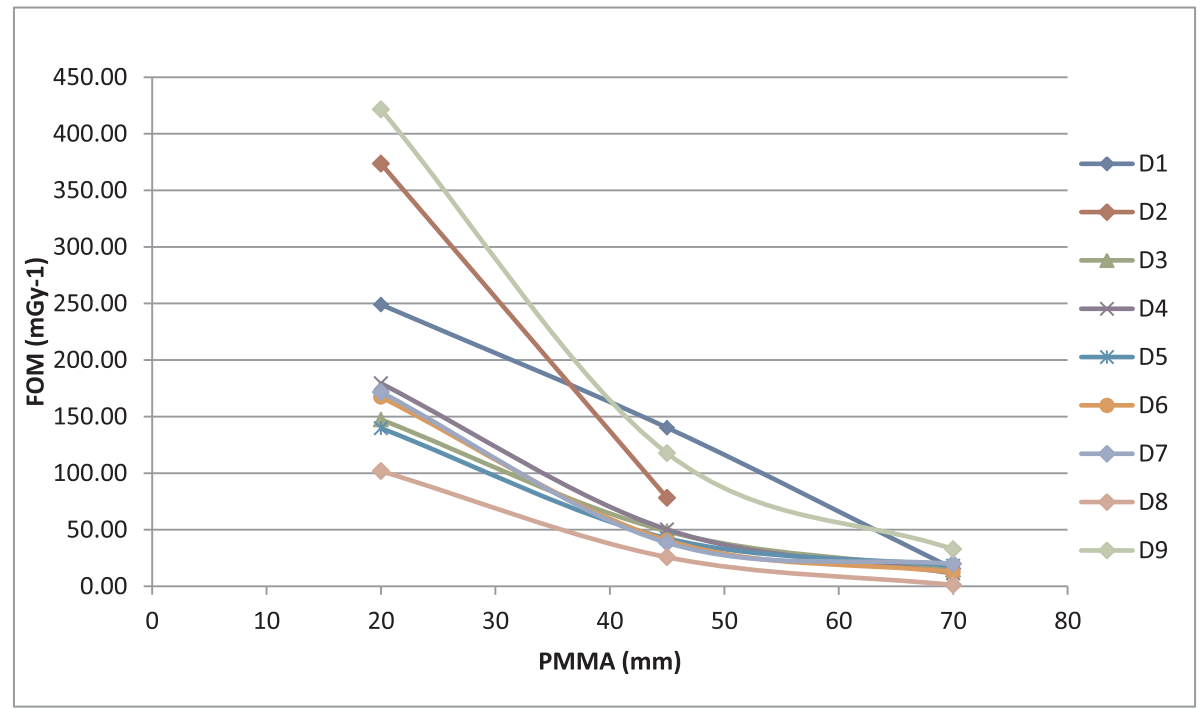

Fig. 4. Figure of Merit for 20, 45 and 70 mm PMMA.

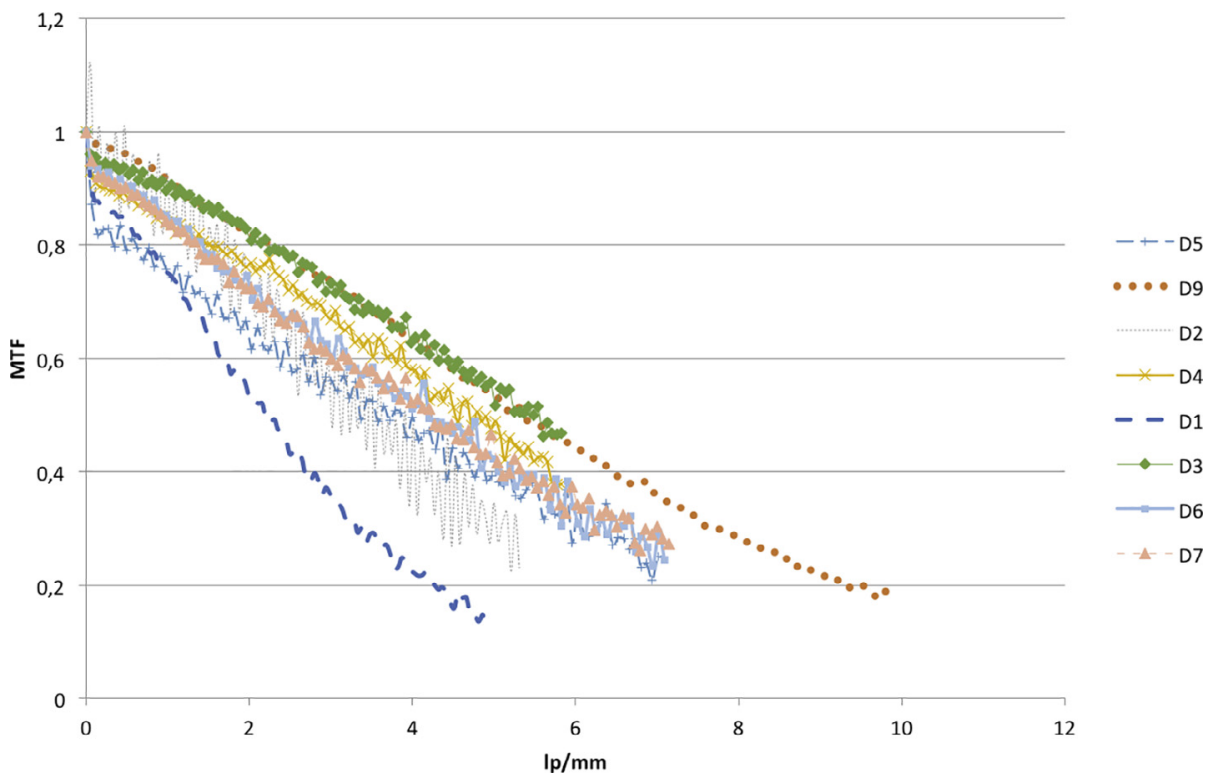

Fig. 5. Modulation transfer function. 
Gold thickness step

$\begin{array}{llllllllllllll}\text { D1 } & 1 & 2 & 3 & 4 & 5 & 6 & 7 & 8 & 9 & 10 & 11 & 12 & 13\end{array}$

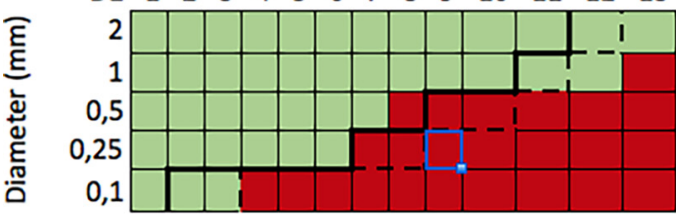

Gold thickness step

$\begin{array}{llllllllllllll}\text { D2 } & 1 & 2 & 3 & 4 & 5 & 6 & 7 & 8 & 9 & 10 & 11 & 12 & 13\end{array}$

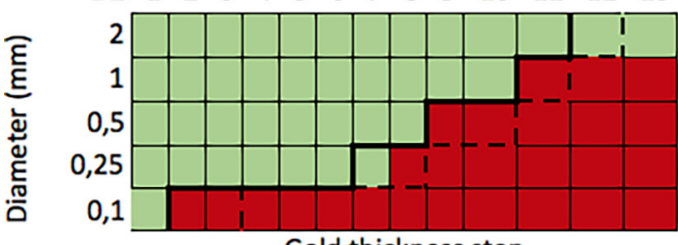

Gold thickness step

$\begin{array}{llllllllllllll}\text { D3 } & 1 & 2 & 3 & 4 & 5 & 6 & 7 & 8 & 9 & 10 & 11 & 12 & 13\end{array}$

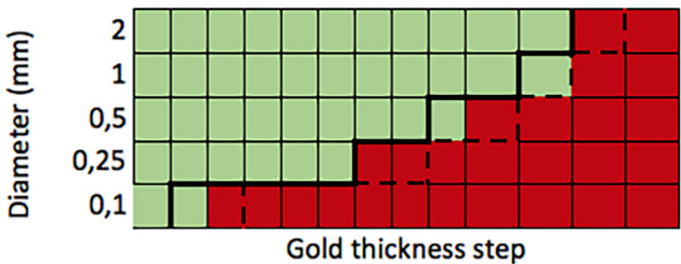

$\begin{array}{llllllllllllll}\text { D4 } & 1 & 2 & 3 & 4 & 5 & 6 & 7 & 8 & 9 & 10 & 11 & 12 & 13\end{array}$

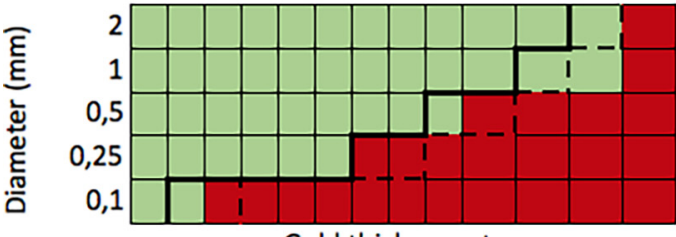

Gold thickness step

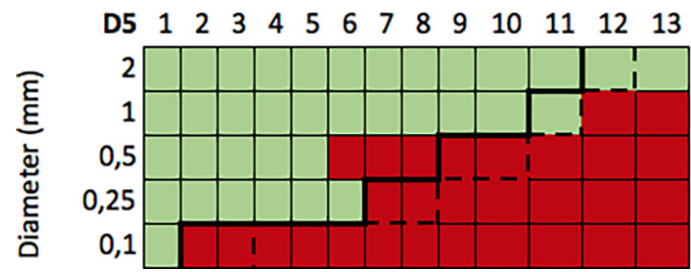

Gold thickness step

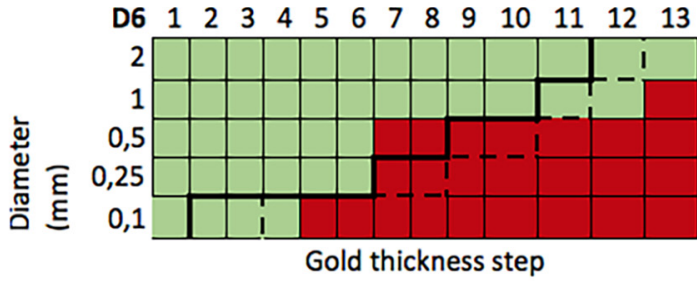

$\begin{array}{llllllllllllll}\text { D7 } & 1 & 2 & 3 & 4 & 5 & 6 & 7 & 8 & 9 & 10 & 11 & 12 & 13\end{array}$

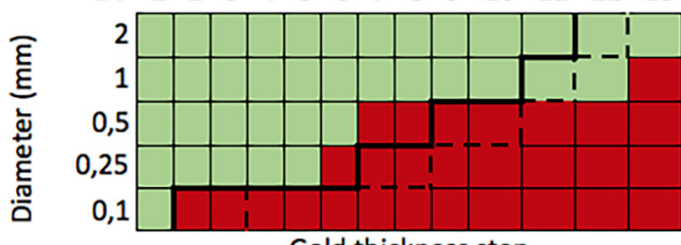

Gold thickness step

$\begin{array}{llllllllllllll}\text { D8 } & 1 & 2 & 3 & 4 & 5 & 6 & 7 & 8 & 9 & 10 & 11 & 12 & 13\end{array}$

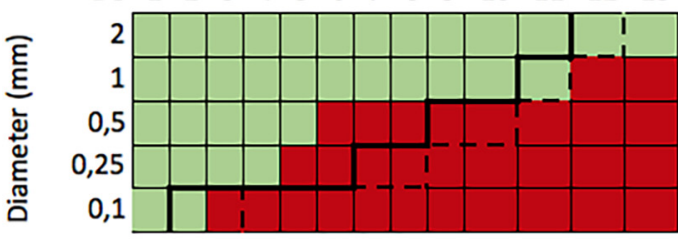

Gold thickness step

$\begin{array}{llllllllllllll}\text { D9 } & 1 & 2 & 3 & 4 & 5 & 6 & 7 & 8 & 9 & 10 & 11 & 12 & 13\end{array}$

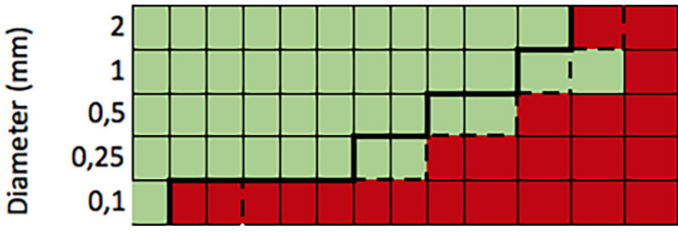

were trained in quality control programs.

- Equipment A1 that performed poorly at the beginning was replaced.

- Equipment D3 even though it passed all QC tests, its detector was changed and overall image quality was improved.

- Equipment D5 had its detector changed and subsequently passed all tests.

- Equipment D6 had service intervention and issues in thickness accuracy and HVL were corrected.

- Equipment D7 showed artefacts with W/Ag combination while imaging thick breast and hence the Ag filter was replaced to improve overall image quality.

\section{Conclusions}

Improvement in terms of quality practices for mammography has commenced in some countries as a result of the medical physics activities of the CRP. Prior to the implementation of the CRP mammography QC programs among participating countries varied significantly. Analogue QC has been done for many years but the transition to digital mammography imposes new aspects and important challenges in the establishment of a complete QC program. Image quality metrics were the ones that showed the need for more attention, advocating the need for comprehensive quality assessment, rather than fragmented efforts of sporadic performance testing.

Human resources, especially concerning medical physicists, were limited at the beginning of the CRP, and in some countries, it was difficult to find and involve medical physicists. Various centres have addressed this need by training medical physicists from other disciplines within medical physics, with further specialized training on mammography occurring during the course of the CRP. Among the group, the radiologists' understanding of the role and need for medical physicists has improved and the involvement of physicists in multidisciplinary teams has been strengthened. Radiographic QC practices in mammography were improved. Furthermore, as an additional benefit to the human resources and infrastructure improvement that resulted out of the CRP, networking among medical physicists and radiologists took place and was maintained over time at the national and international level.

The use of IAEA QC protocols provided a uniformed approach to QC measurements. The spreadsheet developed for the project allowed an adequate collection of data, as well as, a review against tolerances in a more expeditious and validated way. Centralized image analysis using computational tools also allowed greater uniformity of methodology and objectivity in results. 
Regarding QC test results, accessing "raw" images proved to be a challenge at the beginning, while compliance with the limiting values has been an issue in some centres that was addressed through immediate corrective actions. It must be noted that the primary aim of mammography is to provide diagnostically adequate image quality for the purposes of the examination, and radiation protection has to be considered only when this requirement is met. However, the lack of medical physicist who can properly evaluate the image quality, in combination with a strong regulatory emphasis on radiation protection of patients, as reported in other studies [19], create a framework of very tightly supervised radiation dose in isolation from image quality, jeopardizing the diagnostic findings. Medical physicists and service personnel need to conduct optimization actions where doses can be increased to obtain better overall image quality and radiologists know that their subjective evaluation of image quality alone, is not enough to provide the optimum service to their patients.

Another important issue pointed out was that many countries do not have much experience on how to fix discrepancies outside routine service protocols; and many do not have a preventive maintenance contract for their mammography units.

Some of the elements of quality control that are presented in this study were completely missing in certain participating institutes and the application of more comprehensive testing methodologies provided an additional tool for improvement of the mammography QC practice. The results of this study represent a valuable confirmation of the need for comprehensive quality assurance programs in mammography, but also in diagnostic radiology. Sporadic performance testing of a limited number of parameters, such as $\mathrm{kVp}$ and HVL, sometimes only as part of the licensing process, are no longer adequate to investigate in depth the performance of modern mammography systems and point out possible improvements. The active involvement of the medical physicist in the design and the application of such QA programs is mandatory to decrypt all the information that can be derived from the QC testing, bringing added value to the clinical use of the systems and facilitating improved care for the patients.

\section{Acknowledgements}

Jerry Soto, Centro de Investigación en Ciencias Atómicas, Nucleares y Moleculares, Universidad de Costa Rica, Costa Rica.

Manindra Bhushan, Rajiv Gandhi Cancer Institute and Research Centre, Delhi, India.

Eduardo López-Pineda and César Ruiz-Trejo; Instituto de Física, Universidad Nacional Autónoma de México, Ciudad de México, México.

Sidrah Mahmood and Muhammad Arif, Multan Institute of Nuclear Medicine and Radiotherapy, Multan, Pakistan.

Zusana Ćosić, Nebojša Vulin, Igor Janković and Marko Arežina; University Clinical Centre of the Republic of Srpska, Department of
Clinical Radiology, Banja Luka, Bosnia and Herzegovina.

CRP participants are thankful to our CRP coordinator, Dr. Ravi Kashyap, from the Nuclear Medicine and Diagnostic Imaging Section (NMDI) of the Division of Human Health at the International Atomic Energy Agency (IAEA) who passed away during the course of the project. His enthusiasm and motivation moved the entire group in achieving our initial goals.

Spreadsheets used during this CRP are available to all Medical Physicists, please request to corresponding author.

\section{References}

[1] http://globocan.iarc.fr/Default.aspx; 2017 [accessed 9.1.2017].

[2] International Atomic Energy Agency, Quality Assurance Programme for Digital Mammography, IAEA Human Health Series No. 17, IAEA, Vienna; 2011.

[3] CEC Commission of the European Communities. European guideline for quality assurance in breast screening and diagnosis. Report EUR 14821 4th ed European Commission. Office for Official Publications of the European Communities. Luxembourg; 2006.

[4] International Atomic Energy Agency, Quality Assurance Programme for Screen Film Mammography, IAEA Human Health Series No. 2, IAEA, Vienna; 2009.

[5] http://www.leedstestobjects.com/index.php/phantom/tor-max/; 2017 [accessed 2. 3.2017].

[6] http://www.artinis.com/cdmam-40; 2017 [accessed 2.3.2017].

[7] http://www.euref.org/european-Guidelines; 2017 [accessed 2.3.2017] .

[8] http://www.leedstestobjects.com/index.php/phantom/dmam2-gold-phantom/; 2017 [accessed 2.3.2017].

[9] Leeds Test Objects, AutoPIA version 3.2.4, http://autopia.cyberqual.it.

[10] Gennaro G, Ferro F, Contento G, Fornasin F, di Maggio C. Automated analysis of phantom images for the evaluation of long-term reproducibility in digital mammography. Phys Med Biol 2007;52:1387-407.

[11] Rose A. The sensitivity performance of the human eye on an absolute scale. J Opt Soc Am 1948;38:196-208.

[12] Gennaro G, Ballaminut A, Contento G. A multi-parametric, automatic method to monitor long-term reproducibility in digital mammography: results from a regional screening program. Eur Radiol 2017;27(9):3776-87. http://dx.doi.org/10.1007/ s00330-017-4735-x.

[13] Mora P, Blanco S, Khoury H, Leyton F, Cardenas J, Defaz M, et al. Latin American dose survey results in mammography studies under IAEA programme: radiological protection of patients in medical exposures (TSA3). Radiat Prot Dosimetry 2015;163(4):473-9. http://dx.doi.org/10.1093/rpd/ncu205.

[14] International Atomic Energy Agency, Dosimetry in Diagnostic Radiology: An International Code of Practice, Technical Reports Series No. 457, IAEA, Vienna; 2007.

[15] http://www.medphys.it/down_dqe.htm; 2017 [accesed 9.1.2017].

[16] Donini B, Rivetti S, Lanconelli N, Bertolini M. Free software for performing physical analysis of systems for digital radiography and mammography, Med Phys 2014; Vol. 41(5), 051903. doi: 10.1118/1.4870955.

[17] Dance D, Skinner CL, Young KC, Beckett JR, Kotre CR. Additional factors for the estimation of mean glandular breast dose using the UK mammography dosimetry protocol. Phys Med Biol 2000;45(11):3225-40.

[18] Borg M, Badr I, Royle GJ. The use of a figure-of-merit (FOM) for Optimization in digital mammography: a literature review. Radiat Prot Dosimetry 2012;151(1):81-8. org/10.1093/rpd/ncr465.

[19] Mora P, Khoury H, Bitelli R, Quintero AR, Garay F, Aguilar JG, et al. Latin American image quality survey in digital Mammography studies. Radiat Prot Dosimetry 2016;174(1):94-101. http://dx.doi.org/10.1093/rpd/ncw049. 